work is required as less than half of the patients had entries on the Electronic Palliative Care Coordination System and only $6 \%$ had documented evidence of their wishes. Feedback - such as 'All care and treatment have been excellent.' (Patient) and 'Much better links between heart failure team and palliative care team with great benefits for patients' (Staff) - indicates that this collaborative project has been a positive experience, enabling more patients and families to access hospice and other end-of-life care services in their locality.

\section{P-183 ADDRESSING INEQUALITY IN PALLIATIVE CARE PROVISION IN HEART FAILURE}

${ }^{1}$ Karen Chumbley, 'Beata Kasznicka, 'Sue O'Neill, 'Wendy West, ${ }^{2}$ Ness Andrews. ${ }^{1} S t$ Helena, Colchester, UK; ${ }^{2}$ Anglian Community Enterprise, Colchester, UK

\subsection{6/bmispcare-2019-HUKNC.205}

Background There is an urgent need to address the inequality in palliative care provision for people living with heart failure.

North East Essex has a changing demographic, with a predicted $25 \%$ increase in the population over 76 years old (Joint Strategic Needs Assessment North East Essex, 2013). Heart failure is a common life limiting condition affecting more than $10 \%$ of those over 75 years (Mosterd, Hoes, de Bruyne, Deckers et al., 1999). Therefore the number of people living with heart failure in our community is likely to rise significantly. People with heart failure are under-represented on palliative care registers (Gadoud, Kane, Macleod, Ansell et al., 2014). Only 4\% of referrals to St Helena in 2016/17 were for people with heart failure.

Aim To begin to address the inequity of palliative care provision to local people living with advanced heart failure by: increasing referrals to the hospice for people with heart failure; improving access to the local electronic palliative care coordination system (EPaCCS); delivering a coordinated approach between community heart failure services and the hospice and improving knowledge of palliative care in heart failure across both services.

Method We allocated medical time to heart failure service coordination and created a day centre group specifically for people with heart failure. We created a heart failure link nurse role and hosted shared education events on heart failure and palliative care between the hospital, community and hospice services. We developed an inpatient diuresis protocol and audit and created a heart failure and advance care planning animation.

Results Referrals to the hospice increased from 86 to 142 per year, the number of people with heart disease on EPaCCs increased from 136 to 323 and the number of inpatient admissions for people with heart failure increased from 11 to 21.

Conclusion A collaborative focus on a population with a specific diagnosis can begin to address inequality in palliative care provision.

\section{P-184 INITIATING A HOSPICE INPATIENT DIURESIS SERVICE}

Beata Kasznicka, Karen Chumbley, Emma Tempest. St Helena, Colchester, UK
Background Heart failure is a progressive, highly symptomatic condition (McIlvennan \& Allen, 2016), characterised by weakening of the heart muscle, resulting in the inability to maintain an adequate cardiac output. The impact of heart failure is significant with both patients and families requiring much support. One of the typical symptoms is peripheral and pulmonary oedema, which has huge impact on quality of life. This can be managed via parenteral administration of diuretics.

The need for a service offering diuresis in a non-acute setting was identified via multidisciplinary team meetings involving the hospice and the community heart failure team.

Aim To create a safe and effective diuresis service within the hospice inpatient unit for people with advanced heart failure.

Method The diuresis service was commenced after reviewing current literature and exploring already established services. We developed a policy and procedure for the administration of subcutaneous Furosemide within the hospice setting. This was then reviewed by the medical and nursing team within the hospice to ensure it was both safe and feasible.

Results Since the inception of this service in July 2018, St Helena has offered diuresis to 19 patients. An audit showed that we are following our guideline.

Conclusion It is feasible to establish a diuresis service using the administration of subcutaneous Furosemide in a hospice setting. The introduction of this hospice-led service has extended the possibility of diuresis for patients who are not fit for hospital admission and offered the choice of place of care for patients diagnosed with advanced heart failure. The next step is to evaluate the input of this service on patients' symptoms and quality of life.

\section{P-185 INTEGRATED PALLIATIVE HEART FAILURE SERVICE PILOT}

Alison Bacon. St Catherine's Hospice, Preston, UK

\subsection{6/bmjspcare-2019-HUKNC.207}

Background Patients with heart failure are as likely to be symptomatic with advance care planning needs, however, on average make up only $4 \%$ of hospice and palliative care teams' caseload. An application for a grant for a pilot service was made to St James's Place Charitable Foundation.

Aims

- To provide integrated assessments of patients with a primary diagnosis of heart failure who were felt to be in the last year of life;

- Focus on advance care planning and symptom management;

- Provide clinical education on heart failure and palliative care.

Method Through meetings with relevant staff including hospice, local community and hospital NHS Trusts, we developed a service plan, referral criteria-protocol for a targeted, joint (palliative care physician and Heart Failure Clinical Nurse Specialist) intervention with appropriate onward referral and a 'Palliative Care in Heart Failure' conference.

Results The clinical service ran for 13 months: 41 referrals and completed 35 first assessments. $77 \%$ were seen at home (home or nursing/residential home) reflecting the poor functional status of many of these patients. $86 \%$ of patients had medication changes made at the first assessment. Of the patients seen $85 \%$ had a Preferred Place of Care/Preferred 